\title{
Comparison of three dimensional reconstruction and conventional computer tomography angiography in patients undergoing zero-ischemia laparoscopic partial nephrectomy
}

\section{Xiaorong Wu}

Shanghai Jiao Tong University School of Medicine Affiliated Renji Hospital

Chen Jiang

Shanghai Jiao Tong University School of Medicine Affiliated Renji Hospital

\section{Guangyu Wu}

Shanghai Jiao Tong University School of Medicine Affiliated Renji Hospital

Chao Shen

Shanghai Jiao Tong University School of Medicine Affiliated Renji Hospital

Qibo Fu

Shanghai Jiao Tong University School of Medicine Affiliated Renji Hospital

\section{Yonghui Chen}

Shanghai Jiao Tong University School of Medicine Affiliated Renji Hospital

Dongming Liu ( $\square$ rjliudongming@sina.com )

Shanghai Jiao Tong University School of Medicine Affiliated Renji Hospital https://orcid.org/00000002-1883-2281

\section{Wei Xue}

Shanghai Jiao Tong University School of Medicine Affiliated Renji Hospital

\section{Research article}

Keywords: Computer tomography angiography, Laparoscopy, Partial nephrectomy, Three-dimensional reconstruction, Zero ischemia

Posted Date: December 2nd, 2019

DOl: https://doi.org/10.21203/rs.2.17835/v1

License: (c) (1) This work is licensed under a Creative Commons Attribution 4.0 International License. Read Full License 
Version of Record: A version of this preprint was published at BMC Medical Imaging on May 6th, 2020. See the published version at https://doi.org/10.1186/s12880-020-00445-8. 


\section{Abstract}

Background: Advances in the three dimensional (3D) reconstruction and printing technology have fueled a rapidly growing interest in its applications in the field of urology. To our knowledge, the experience on the use of 3D reconstruction to facilitate zero-ischemia partial nephrectomy (PN) remains sparse and the role of $3 \mathrm{D}$ reconstruction is still needed to be defined. This study aimed to assess the role of threedimensional (3D) reconstruction and conventional computer tomography angiography (CTA) in zeroischemia laparoscopic partial nephrectomy (LPN).

Methods: A total of 60 consecutive patients undergoing zero-ischemia LPN between October 2017 and March 2018 underwent CTA (CTA group including 30 patients) and 3D reconstruction (3D group including the remaining 30 patients) were included. 3D reconstruction and CTA images were prepared which were used to demonstrate the number and spatial interrelationships of the location of renal tumors and tumor feeding arteries. These radiology findings were directly correlated with intraoperative surgical findings at laparoscopy. Demographics, perioperative characteristics and renal function were compared between groups.

Results: CTA and 3D reconstruction were successfully performed without any related complications. All the procedures were performed successfully without conversion to main renal artery clamping. Preoperative 3D reconstruction identified that 15 patients had only one tumor feeding artery, 12 had two, and another 3 had three, while the conventional CTA revealed that 22 patients had one tumor feeding artery, 8 had two $(P>0.05)$. The mean operation time was shorter and estimated blood loss was less in the $3 \mathrm{D}$ group $(\mathrm{P}<0.05)$. 3D reconstruction group resulted in more accurate dissection of tumor artery $(91.7 \%)$ as compared to conventional CTA (84.2\%). There were no statistical differences in the baseline characteristics and renal function outcomes between two groups.

Conclusions: 3D reconstruction can provide comprehensive information for the preoperative evaluation and intraoperative orientation that may facilitate tumor resection during zero-ischemia LPN for renal tumors.

\section{Background}

Laparoscopic partial nephrectomy (LPN) has gained popularity as a promising minimally invasive nephron sparing option for clinical T1 renal tumors [1]. Minimizing or even eliminating warm ischemic injury was the aim for improving the functional outcomes of nephron sparing surgery. Recent advances in surgical technique now make it possible to eliminate global ischemia completely during partial nephrectomy (PN), such as zero ischemia PN, unclamped "minimal-margin" PN and clampless techniques [2-4]. However, zero ischemia LPN is a technically challenging operation. As such, a detailed and casespecific understanding of the particular renovascular anatomy is of great importance to surgical planning and even orientation. 
At most centers, renal angiography has been the preferred imaging modality for preoperative evaluation of patients with renal tumors. Although helical computerized tomography (CT) allow high quality images of the renal vasculature and soft tissue anatomy to be obtained in any plane, advances in the three dimentional (3D) reconstruction and printing technology have fueled a rapidly growing interest in its applications in the field of urology [5]. This image modality has been successfully used for preoperative evaluation of patients undergoing PN [6, 7]. Currently, the experience on the use of 3D reconstruction to facilitate zero-ischemia PN remains sparse, and the role of 3D reconstruction is still needed to be defined. We determined whether $3 \mathrm{D}$ reconstruction could be the preferred preoperative radiological examination, providing all information necessary for surgical planning and orientation for zero-ischemia LPN.

\section{Methods}

\section{Patients}

Between January 2018 and July 2018, 60 consecutive patients who underwent zero-ischemia LPN in our hospital were retrospectively analyzed. The patients with sporadic renal tumor at clinical T1NOMO stage were included. Patients with multiple kidney tumors, bilateral kidney tumors, or a solitary kidney tumor were excluded from this study. The study was approved by the Institutional Review Board of Ren Ji Hospital affiliated to School of Medicine of Shanghai Jiao Tong University. Before surgery, all the patients received contrast-enhanced CT with angiography, and additional 3D reconstruction was conducted in 30 patients.

\section{CTA and 3D reconstruction protocol}

All CTA examinations were performed using a 64-multidetector computed tomography scanner (VCT Light Speed, GE Healthcare, Pittsburgh, USA). Patients drank $1000 \mathrm{ml}$ of water before the examination. Four phase images were obtained in a craniocaudal orientation. The scanning covered the area from the diaphragm to the lower kidney poles (non-enhanced, arterial and portal phases) and from the diaphragm to the symphysis pubis (delayed nephrographic excretory phase). Contrast-enhanced images were obtained after intravenous administration of $150 \mathrm{ml}$ of non-ionic contrast medium (Iopamiro, Bracco, Milan, Italy). The current was 110-380 mA, the slice thickness was $1.25-\mathrm{mm}$, and the pitch was 1.375 . Non-enhanced nephrographic and excretory phase scans were reconstructed as $1.25-\mathrm{mm}$ sections. The arterial phase images were reconstructed at an $0.725-\mathrm{mm}$ interval.

Images in DICOM format were processed with a dedicated software by Mimics, 3-matic and Magics (Materialise, Belgium). Then, a 3D virtual model was rendered on the basis of CTA examination. The reconstruction focused on the semitransparent kidney, the tumor characteristics and the arterial vasculature. The segmentation of kidney surface was performed in the arterial phase images with automated threshold segmentation method using Hounsfield units. The renal pedicle and tumor feeding arteries were reconstructed in the arterial phase by using the dynamic region growing method. Then, the virtual renovascular-tumor models were reviewed by both urologists and bioengineers to evaluate the accuracy of models in comparison to the CTA images (Figure.1). The 3D model and the relative 
interactive 3D images were then created. The number of renal tumor feeding arteries was compared between the 2 imaging modalities and matched with intraoperative findings on laparoscopy, which were considered the gold standard.

\section{Surgical technique}

Patients received general anesthesia and lied in a lateral decubitus position. All the procedures were performed using a retroperitoneal approach. Four ports in the lumbar region were applied. In the CTA group, off-clamp LPN was performed with the guidance of CTA images. In the 3D group, snapshots of 3D images from various angle views (e.g., lateral, anterior and posterior) were created and presented to the surgeon preoperatively and intraoperatively. After the tumor was completely exposed, the parenchyma was then incised, and the tumor was excised along its capsule with a margin of 1 to $2-\mathrm{mm}$ normal parenchyma by blunt dissection, the dissection was kept at the relatively avascular intrarenal plane. On reaching the target vessels of the tumor, operation was done with Hem-o-lok clips (Figure.2). Homeostasis was achieved with point-specific suturing, and incised calyces were repaired. Then, the defected parenchyma was closed via continuous suturing with Hem-o-lok clips.

\section{Outcome Measures}

The general information, tumor characteristics including RENAL score [8] and the number of feeding arteries were collected and compared. All complications within 30 days after surgery were considered being related to surgery, and staged according to the 2004 Clavien-Dindo grading system [9]. The estimated glomerular filtration rate (eGFR) was calculated for all patients preoperatively and 12 months postoperatively according to the Modification of Diet in Renal Disease Equation [10]. Statistical analysis was performed using Student t-test for continuous variables or Chi-square for categorical variables, and a value of $\mathrm{P}<0.05$ was considered statistically significance.

\section{Results}

A total of 60 consecutive patients who underwent zero-ischemia LPN were included in this study. Patient demographics and surgical variables are reported in Table 1. The mean tumor size was 4.1 and $3.8 \mathrm{~cm}$ in 3D group and CTA group respectively without statistical significance. There were also no significant differences between two groups in the age, gender, body mass index and RENAL score. 
Table 1

Patients' demographics and surgical variables

\begin{tabular}{|c|c|c|c|}
\hline Variables & $\begin{array}{l}\text { 3D group } \\
\text { (30 cases) }\end{array}$ & $\begin{array}{l}\text { CTA group } \\
\text { (30 cases) }\end{array}$ & $\mathrm{p}$ value \\
\hline Gender, n (\%) & & & 0.774 \\
\hline Male & $22(73.3)$ & $21(70)$ & \\
\hline Female & $8(26.7)$ & $9(30)$ & \\
\hline Age (years, mean $\pm S D$ ) & $57.6 \pm 11.7$ & $56.4 \pm 9.8$ & 0.669 \\
\hline $\mathrm{BMI}\left(\mathrm{kg} / \mathrm{cm}^{2}\right.$, mean $\left.\pm \mathrm{SD}\right)$ & $25.2 \pm 2.8$ & $24.9 \pm 2.5$ & 0.622 \\
\hline Tumor size $(\mathrm{cm}$, mean $\pm \mathrm{SD})$ & $4.1 \pm 0.84$ & $3.8 \pm 0.76$ & 0.082 \\
\hline Renal Score, n (\%) & & & 0.339 \\
\hline $4-6$ & $11(36.6)$ & $12(40)$ & \\
\hline $7-9$ & $17(56.7)$ & $15(50)$ & \\
\hline $10-12$ & $2(6.7)$ & $3(10)$ & \\
\hline Operative time (min, mean \pm SD) & $125.4 \pm 19.7$ & $136.6 \pm 15.1$ & 0.017 \\
\hline $\begin{array}{l}\text { Estimated blood loss } \\
(\mathrm{ml} \text {, mean } \pm \text { SD })\end{array}$ & $130.3 \pm 39.9$ & $179.0 \pm 77.2$ & 0.003 \\
\hline Hospital stay (days, mean \pm SD) & $5.0 \pm 1.1$ & $5.4 \pm 1.0$ & 0.114 \\
\hline Pathologic subtype, n (\%) & & & 0.543 \\
\hline Clear cell & $26(86.7)$ & 25 (83.3) & \\
\hline Chromophobe & $1(3.3)$ & $3(10)$ & \\
\hline Papillary & $3(10)$ & $2(6.7)$ & \\
\hline Furhman grade, $\mathrm{n}(\%)$ & & & 0.740 \\
\hline I & $10(33.3)$ & $12(40)$ & \\
\hline II & $16(53.4)$ & $13(43.3)$ & \\
\hline III & $4(13.3)$ & $5(16.7)$ & \\
\hline Complications, n (\%) & & & 0.365 \\
\hline Fever & 1 (3.3\%) & 0 & \\
\hline Hematuria & $1(3.3)$ & $3(10 \%)$ & \\
\hline
\end{tabular}




\begin{tabular}{|llll|}
\hline Variables & $\begin{array}{l}\text { 3D group } \\
\text { (30 cases) }\end{array}$ & $\begin{array}{l}\text { CTA group } \\
\text { (30 cases) }\end{array}$ & p value \\
\hline $\begin{array}{l}\text { eGFR change } \\
\left(\mathrm{ml} / \mathrm{min} / 1.73 \mathrm{~m}^{2}, \text { mean } \pm \mathrm{SD}\right)\end{array}$ & $-8.67 \pm 8.8$ & $-10.6 \pm 11.5$ & 0.459 \\
\hline
\end{tabular}

The number of tumor feeding arteries was identified preoperatively by 3D reconstruction and CTA, and then confirmed intraoperatively (Table 2). Preoperative 3D reconstruction identified that 15 patients had only one tumor feeding artery, 12 patients had two, and another 3 had three. While conventional CTA revealed that 22 patients had one tumor feeding artery and 8 patients had two, without significant statistical difference as compared to the 3D group. However, under the guidance of 3D reconstruction images, more tumor feeding arteries were correctly identified during the surgery as compared to surgery under the guidance of conventional CTA $(P=0.03)$. Although there was no significant difference between two groups, the rate of accurate tumor feeding artery orientation was higher in the 3D group than in the CTA group $(91.7 \%$ vs $84.2 \%, p=0.285)$.

Table 2

Tumor feeding arteries according to 3D reconstruction of renovascular-tumor, conventional CTA and surgically confirmed findings in 60 patients

\begin{tabular}{|llll|}
\hline Variables & 3D group & CTA group & p value \\
\hline Preoperative findings of tumor feeding arteries, $n(\%)$ & & & 0.077 \\
\hline 1 & $15(50)$ & $22(73.3)$ & \\
\hline 2 & $12(40)$ & $8(26.7)$ & \\
\hline 3 & $3(10)$ & $0(0)$ & 0.03 \\
\hline $\begin{array}{l}\text { No. surgically } \\
\text { confirmed findings, } n(\%)\end{array}$ & & & \\
\hline 0 & $1(3.3)$ & $1(3.3)$ & \\
\hline 1 & $16(53.3)$ & $26(86.7)$ & \\
\hline 2 & $11(36.7)$ & $3(10)$ & 0.285 \\
\hline 3 & $2(6.7)$ & $0(0)$ & \\
\hline Rate of accurately tumor feeding arteries orientation & $91.7 \%(44 / 48)$ & $84.2 \%(32 / 38)$ & \\
\hline
\end{tabular}

Zero-ischemia LPN was successfully performed in all the patients without conversion to main renal artery clamping, open surgery or radical nephrectomy. The mean operation time (OT) was $125.4 \pm 19.7$ min and $136.6 \pm 15.1 \mathrm{~min}$ in the 3D group and CTA group, respectively $(P=0.017)$. And the average estimated blood loss (EBL) was significantly less in the 3D group as compared to the CTA group $(P=0.003)$. 
However, no statistical differences were found between two groups in the hospital stay, major surgical complications and change in mean eGFRs (Table 1).

Histopathological results showed clear cell renal cell carcinoma (RCC) (3D group: $n=26$; CTA group: $n=$ 25), chromophobe RCC (3D group: $n=1$; CTA group: $n=3$ ), and papillary RCC (3D group: $n=3$; CTA group: $n=2)$. There were no significant differences in the subtypes and Furhman grade between the two groups $(P>0.05)$. The surgical margins were all negative, and no renal capsule invasion was found. The median duration of follow-up was 18 months in the subgroups, and no local recurrence or metastasis was found.

\section{Discussion}

During conventional LPN, renal artery clamping could provide good intraoperative visualization and bleeding control, but inevitably causes warm ischemia injury [11]. Warm ischemia injury remains one of the most important factors influencing postoperative renal function during nephron sparing surgery. Several techniques have been developed to preserve better postoperative renal function, such as segmental artery clamping and thermal ablation techniques [12, 13].

The concept of zero-ischemia LPN was firstly introduced by Gill et al. [14]. They tried to eliminate global renal ischemia by meticulous microdissection of tertiary or quaternary renal arterial branches feeding the tumor, which is based on the concept of anatomical renovascular microdissection [3]. However, dissecting tumor feeding arteries from the renal hilum is technically difficult and time-consuming. Although off-clamp LPN has been developing and popularizing among the urologic communities $[15,16]$, the uncertainty of the intrarenal relationships of the tumor and feeding arteries makes off-clamp partial nephrectomy even more difficult. Therefore, preoperative understanding of the tumor and renal vascular anatomy is of great importance.

Preoperative CTA has been the preferred imaging modality for the evaluation of renal tumors in our hospital. With the development of CT and computer technology, it allows the generation of high quality images of the renal vasculature, tumor and collecting system at any plane [17]. It can also facilitate establishing conventional 3D models to visualize the arterial vasculature within the hilum, guiding in choosing the appropriate hilar approach and intraoperative target orientation during segmental artery clamping [18]. However, conventional CTA has a poor capacity to display the precise location and the precise anatomical interrelationship of various intrarenal tributaries, especially the tumor feeding arteries. Moreover, conventional 3D models based on CTA typically display kidney, renal vascular and tumor as opaque, which makes it impossible to visualize the intrarenal relationships between the tumor and its adjacent feeding arteries [6]. As a well performed preoperative evaluation of the relevant intrarenal anatomy and intraoperative orientation of tumor feeding arteries is needed for zero-ischemia LPN, it is imperative to develop new techniques for radiologic guidance.

Conventional 3D CT reconstruction techniques typically present kidney, tumor and renal vessels as opaque, which makes it impossible to visualize the intrarenal relationships of the tumor and adjacent feeding arteries [6]. In this study, the patients in the 3D group underwent 3D reconstruction. The 
reconstruction images included 3D surface rendered semitransparent renal tumor, transparent kidney, and 3D course of extra- and intrarenal tumor feeding arteries (Figure.1C-D). Therefore, the location and number of tumor feeding arteries were clarified much more easily. In addition, the use of 3D reconstruction of renovascular tumor resulted in more accurate dissection of target tumor feeding artery than in the CTA group during the operation, thus decreasing intraoperative OT and EBL.

When compared to conventional CTA, 3D reconstruction techniques have several advantages. It could clearly display the intrarenal arterial tree and the detailed anatomical relationships between the tumor, collecting system and intrarenal arteries. The reconstructed images can also be rotated to clarify the number and location of tumor feeding arteries. It can not only provide reference for the preoperative evaluation and determination of appropriate dissection strategy, but also for the intraoperative orientation, therefore avoiding invisible injury to other interlobar arteries during zero-ischemia LPN.

There were also several limitations in this study. First, this was a single-center retrospective study with small sample size and patients received only short-term follow up, the evaluation of long-term outcomes is still awaited. Second, the mean tumor size was 4.1 and $3.8 \mathrm{~cm}$ in each subgroup, whether this approach is also applicable in the management of $\mathrm{T} 1 \mathrm{~b}$ renal tumors is still unclear. Robot assisted zeroischemia partial nephrectomy may be helpful for better tumor resection, renorrhaphy and renal function preservation in the management of T1b or even complicated renal tumors $[4,19]$, but our experience is also lacking in this field. Third, the 3D reconstruction technique based on CT images is not applicable to magnetic resonance imaging. Thus, patients who cannot receive enhanced CT or CTA are excluded from the study. Although the tumor feeding arteries were confirmed more accurately under the guidance of 3D reconstruction technique, the rate of accurately tumor feeding arteries orientation was only $91.7 \%$, without statistical significance as compared with conventional CTA technique.

\section{Conclusions}

Our study indicates 3D reconstruction technique might be a useful tool, which can provide comprehensive information of intrarenal interrelationships between renal tumor and its feeding arteries. The 3D model seems to facilitate tumor feeding artery orientation and dissection, therefore improving the surgical outcome after zero-ischemia LPN.

\section{Declarations}

\section{List of abbreviations}

3D: three dimensional; CT: computerized tomography; CTA: computer tomography angiography; EBL: estimated blood loss; eGFR: estimated glomerular filtration rate; LPN: laparoscopic partial nephrectomy; OT: operation time; PN: partial nephrectomy; RCC: renal cell carcinoma 


\section{Ethical approval and consent to participate}

This study was approved by our Institutional review board (Shanghai Jiao Tong University School of Medicine affiliated Ren Ji Hospital, Shanghai, China) and patients' consent to participate was obtained.

\section{Consent for publication}

Not applicable.

\section{Availability of data and materials}

All data are fully available upon reasonable request. The corresponding author should be contacted if someone wants to request the data.

\section{Competing interests}

The authors declare no competing interests.

\section{Funding}

This study was funded by the Three-year-action-project of Shanghai Hospital Development centre (No: 16CR3062B), the Medical-Engineering cross fund of Shanghai Jiao Tong University (No: YG2017QN46) and the Doctorial Innovation Fund of School of Medicine, Shanghai Jiao Tong University (No: CBXJ201804).

\section{Authors' contributions}

Study concepts and design: XW, CJ, DL and WX; literature research and data collection: XW, CJ, GW, CS, QF and YC; Stastical analysis: CS, QF and YC; manuscript preparation: XW, CJ, GW and CS; manuscript editing: DL and WX. All authors have read and approved the manuscript.

\section{Acknowledgements}

None. 


\section{Author details}

${ }^{1}$ Department of Urology, Shanghai Jiao Tong University School of Medicine affiliated Ren Ji Hospital, 160 Pu Jian Road, 200127, Shanghai, People's Republic of China.

${ }^{2}$ Department of Radiology, Shanghai Jiao Tong University School of Medicine affiliated Ren Ji Hospital, 160 Pu Jian Road, 200127, Shanghai, People's Republic of China.

\section{References}

1.

Salagierski M, Wojciechowska A, Zając K, Klatte T, Thompson RH, Cadeddu JA, et al. The Role of Ablation and Minimally Invasive Techniques in the Management of Small Renal Masses. Eur Urol Oncol. 2018;1(5):395-402.

2.

Li Y, Zhou L, Bian T, Xiang Z, Xu Y, Zhu Y, et al. The zero ischemia index (ZII): a novel criterion for predicting complexity and outcomes of off-clamp partial nephrectomy. World J Urol. 2017;35(7):1095102.

3.

Satkunasivam R, Tsai S, Syan S, Bernhard JC, de Castro Abreu AL, Chopra S, et al. Robotic Unclamped "Minimal-margin" Partial Nephrectomy: Ongoing Refinement of the Anatomic Zero-ischemia Concept. Eur Urol. 2015;68(4):705-12.

4.

Bertolo R, Simone G, Garisto J, Nakhoul G, Armanyous S, Agudelo J, et al. Off-clamp vs on-clamp robotic partial nephrectomy: Perioperative, functional and oncological outcomes from a propensity-score matching between two high-volume centers. Eur J Surg Oncol. 2019;45(7):1232-7.

5.

Youssef RF, Spradling K, Yoon R, Dolan B, Chamberlin J, Okhunov Z, et al. Applications of threedimensional printing technology in urological practice. BJU Int. 2015;116(5):697-702.

6.

Ukimura O, Nakamoto M, Gill IS. Three-Dimensional Reconstruction of Renovascular-Tumor Anatomy to Facilitate Zero-Ischemia Partial Nephrectomy. Eur Urol. 2012;61(1):211-7.

7.

Porpiglia F, Bertolo R, Checcucci E, Amparore D, Autorino R, Dasgupta P, et al. Development and validation of 3D printed virtual models for robot-assisted radical prostatectomy and partial nephrectomy: urologists' and patients' perception. World J Urol. 2018;36(2):201-7.

8.

Kutikov A, Uzzo RG. The R.E.N.A.L. nephrometry score: a comprehensive standardized system for quantitating renal tumor size, location and depth. J Urol. 2009;182(3):844-53.

9. 
Dindo D, Demartines N, Clavien PA. Classification of surgical complications: a new proposal with evaluation in a cohort of 6336 patients and results of a survey. Ann Surg. 2004;240(2):205-13. 10 .

Levey AS, Stevens LA, Schmid CH, Zhang YL, Castro AF 3rd, Feldman HI, et al. A new equation to estimate glomerular filtration rate. Ann Intern Med. 2009;150(9):604-12.

11.

Lieberman L, Barod R, Dalela D, Diaz-Insua M, Abaza R, Adshead J, et al. Use of Main Renal Artery Clamping Predominates Over Minimal Clamping Techniques During Robotic Partial Nephrectomy for Complex Tumors. J Endourol. 2017;31(2):149-52.

12.

Shao P, Tang L, Li P, Xu Y, Qin C, Cao Q, et al. Application of a vasculature model and standardization of the renal hilar approach in laparoscopic partial nephrectomy for precise segmental artery clamping. Eur Urol. 2013;63(6):1072-81.

13.

Huang J, Zhang J, Wang Y, Kong W, Xue W, Liu D, et al. Comparing Zero Ischemia Laparoscopic Radiofrequency Ablation Assisted Tumor Enucleation and Laparoscopic Partial Nephrectomy for Clinical T1a Renal Tumor: A Randomized Clinical Trial. J Urol. 2016;195(6):1677-83.

14.

Gill IS, Eisenberg MS, Aron M, Berger A, Ukimura O, Patil MB, et al. "Zero ischemia" partial nephrectomy: novel laparoscopic and robotic technique. Eur Urol. 2011;59(1):128-34.

15.

Anderson BG, Potretzke AM, Du K, Vetter JM, Bergeron K, Paradis AG, et al. Comparing Off-clamp and Onclamp Robot-assisted Partial Nephrectomy: A Prospective Randomized Trial. Urology. 2019;126:102-9. 16.

Simone G, Tuderti G, Anceschi U, Ferriero M, Costantini M, Minisola F, et al. "Ride the Green Light": Indocyanine Green-marked Off-clamp Robotic Partial Nephrectomy for Totally Endophytic Renal Masses. Eur Urol. 2019;75(6):1008-14.

17.

Shao P, Tang L, Li P, Xu Y, Qin C, Cao Q, et al. Precise segmental renal artery clamping under the guidance of dual-source computed tomography angiography during laparoscopic partial nephrectomy. Eur Urol. 2012;62(6):1001-8.

18.

Shao P, Li P, Xu Y, Cao Q, Ju X, Qin C, et al. Application of combined computed tomography arteriography, venography, and urography in laparoscopic partial nephrectomy with segmental artery clamping. Urology. 2014;84(6):1361-5.

19.

Antonelli A, Veccia A, Francavilla S, Bertolo R, Bove P, Hampton LJ, et al. On-clamp versus off-clamp robotic partial nephrectomy: A systematic review and meta-analysis. Urologia. 2019;86(2):52-62.

\section{Figures}




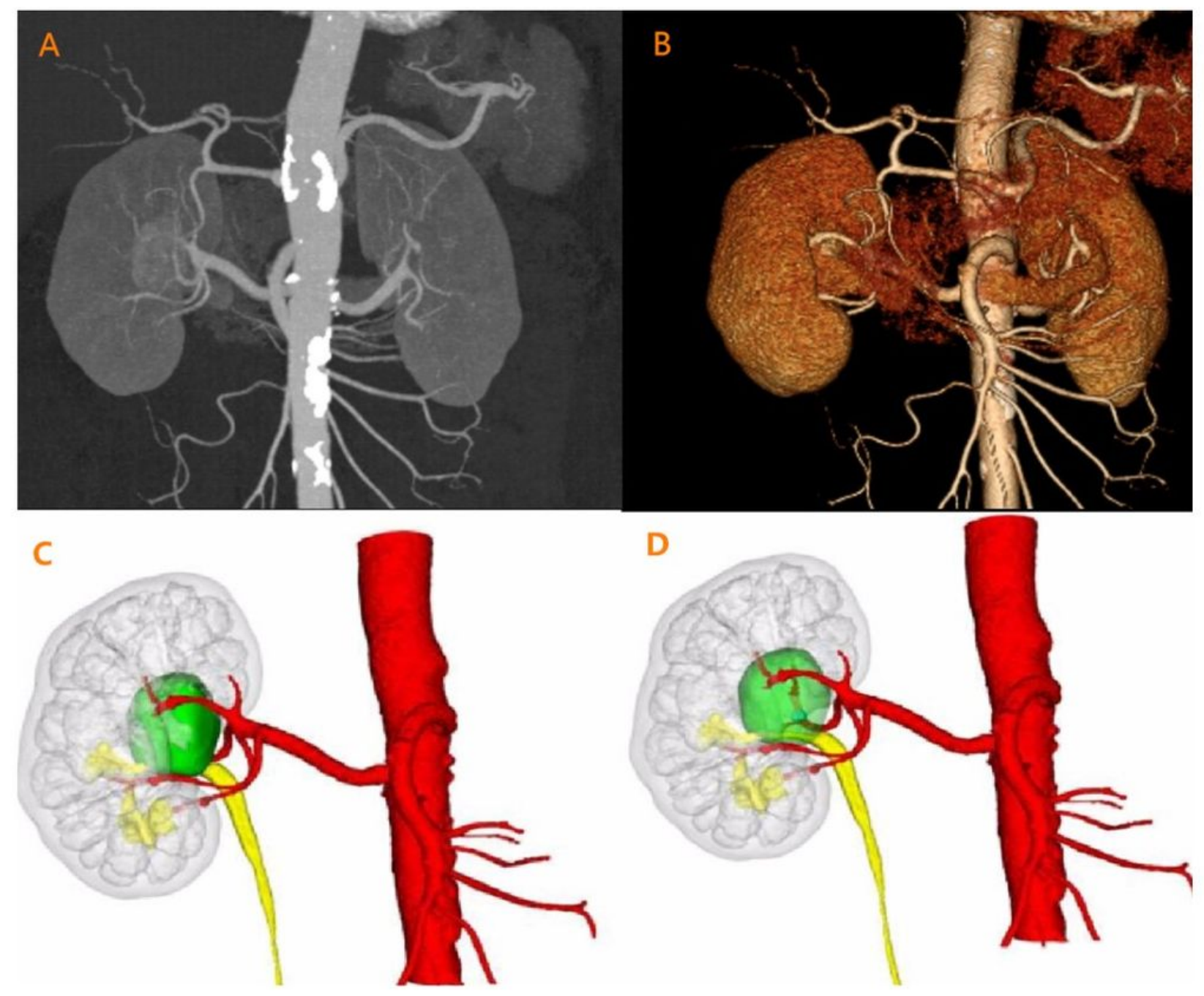

\section{Figure 1}

Conventional CTA and 3D reconstruction demonstrated a tumor located at the renal hilum. A:

Conventional CTA image could identify extrarenal arteries and the tumor, and sometimes the intrarenal artery branches, but failed to illustrate the relationship between tumor and its feeding arteries. B:

Conventional 3D reconstruction based on the CTA could reveal the relationship between kidney, tumor and extrarenal arteries, but it typically present kidney, tumor and renal vessels as opaque, which makes it impossible to visualize the intrarenal antomy and tumor feeding arteries. C-D: The 3D reconstruction images in our study included transparent kidney and 3D course of extra- and intrarenal tumor feeding arteries (C) and 3D surface rendered semitransparent renal tumor, which make it possible to show the targer feeding arteries all around the tumor (D). 


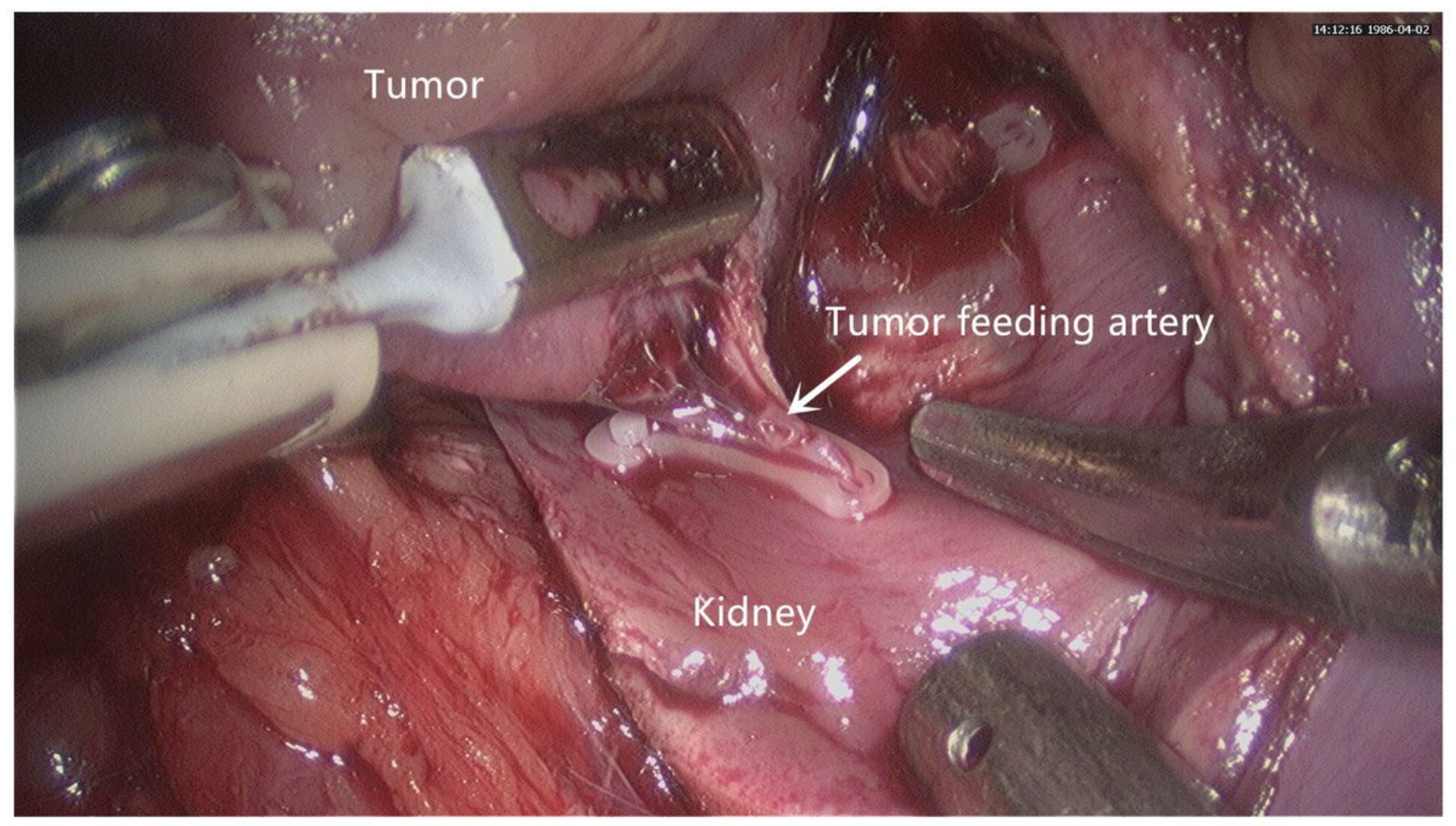

\section{Figure 2}

The tumor was excised with blunt dissection along its capsule. Under the guidance of 3D reconstructed images, the tumor specific feeding artery (white arrow) could be identified and then clamped with the Hem-o-lok clips during the operation. 